\title{
Sphenochoanal Polyp - "The Forgotten Element?"
}

https://doi.org/10.47210/bjohns.2021.v29i2.371

Misbahul Haque, ${ }^{1}$ Subhrajit Das, ${ }^{1}$ Subrata Mukhopadhyay ${ }^{1}$

\section{Introduction}

\section{ABSTRACT}

The occurrence of isolated spheno-choanal polyps are not very common and can be very well confused with antro-choanal polyps or adenoids in adolescents and children. Appropriate diagnosis and prompt surgical intervention are essential for its removal.

Case Report

We report one such case of a 16 year old female who presented with complaints nasal obstruction and reduced hearing. Endoscopic polypectomy was done with complete removal of the polyp.

Discussion

Sphenochoanal Polyp is often misdiagnosed and proper investigations are thus essential for its diagnosis. Surgical Approach is the mainstay form of treatment with complete excision of the polyp.

$\underline{\text { Keywords }}$

Nasal Polyps, Sphenochoanal

$\mathrm{N}$ asal polyps are hypertrophied edematous mucosa of the nasal cavity and paranasal sinuses. They can be Antro-choanal (originating from maxillary antrum), Ethmo-choanal(originating from the ethmoidal sinus) or Sphenochoanal (originating from the sphenoid sinus). These polyp have one part in the sinus also called the intra-sinusal part, another part near the sinus ostium also called the ostial part, and the part outside the sinus is called the extra-sinusal portion. Sphenochoanal polyps rarely occur but recently, various reports have surfaced up.

\section{Case Report}

A 16 year old girl presented to the outdoor with complaints of Nasal obstruction since 1 year which was insidious in onset and was more on the right side than the left. This was also associated with reduced sense of smell and reduced hearing for 8 months. There was no instance of pain, bleeding or any sign of neural involvement. She has been having episodes of nasal discharge and sneezing frequently for 1-2 years which reduced spontaneously most often and sometimes with medications.
Initial impressions with this patient, by taking consideration of history and age, pointed provisionally towards a diagnosis of adenoids. On examination, anterior rhinoscopy was within normal limits. Posterior rhinoscopy revealed a smooth pale mass occluding the right lateral wall of nasopharynx and right choana.

Patient was scheduled for a Diagnostic Nasal endoscopy preceded by CT scan (Fig. 1). CT scan revealed a homogenous mass involving the sphenoid sinus, right lateral wall of nasopharynx and the right choana with some portion in the right nasal cavity along with collection in the left maxillary sinus. According to the study done by Hiremath et. $\mathrm{al}^{1}$, the pneumatization of sphenoid sinus has a very important role in planning the surgery. Walls of Sphenoid sinus are surrounded by important vital structures viz. Internal carotid artery, optic nerve, pituitary gland etc. and hence knowing

1 - Department of ENT, Medical College, Kolkata

Corresponding author:

Dr Misbahul Haque

email: doctor.misbahul@gmail.com 


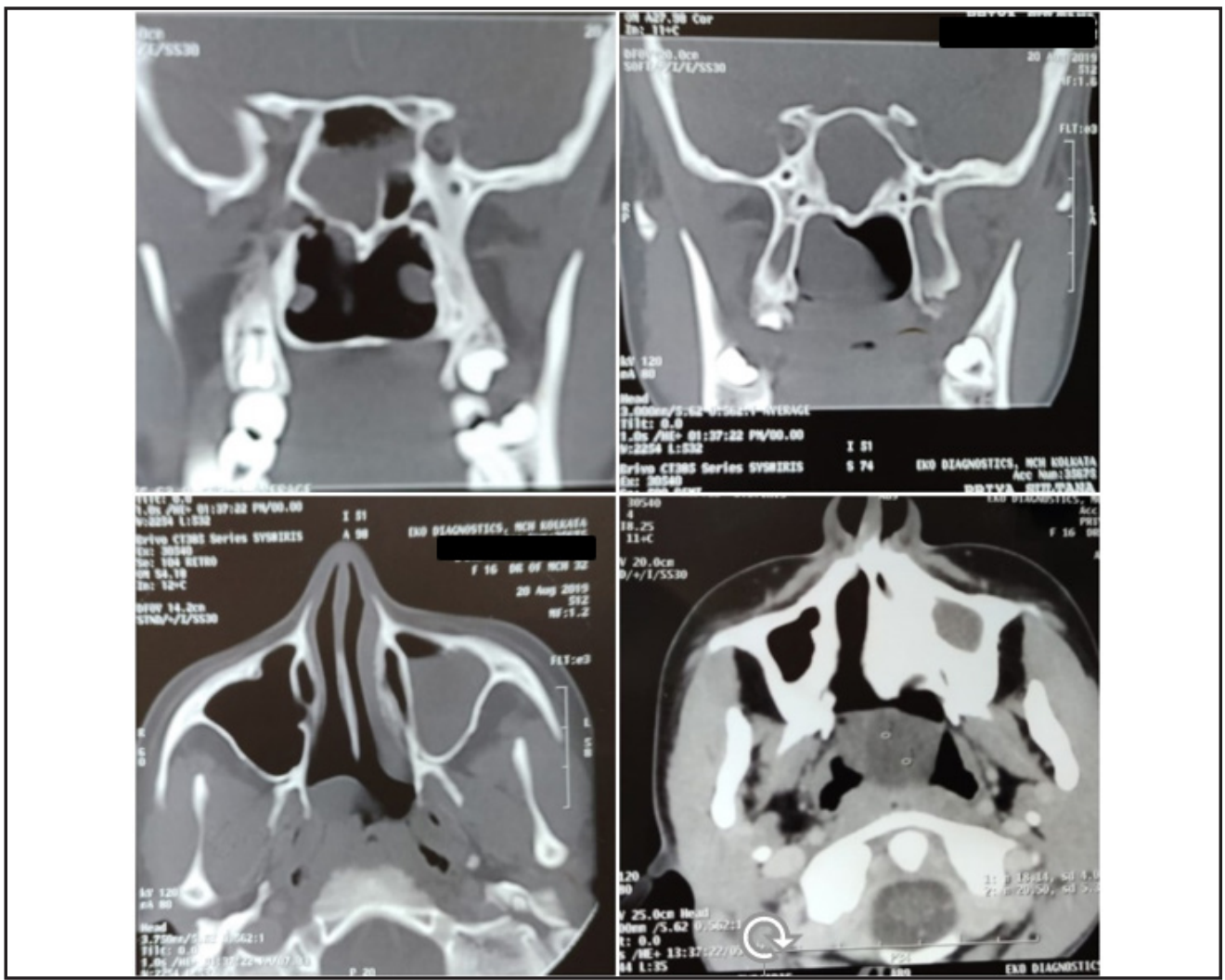

Fig. 1. CT scan showing Sphenochoanal polyp involving the sphenoid sinus and right side of lateral wall of nasopharynx. Also note, the right maxillary sinus is disease free. Small collection noted in left maxillary sinus

the pneumatization of sphenoid sinus pre-operatively can give a clear idea about the necessary planning and approach towards surgery. Extensive pneumatization like sellar type or even lateral extension of sphenoid sinus can result in injury to the vital structures mentioned above and can cause devastating complications. In this case the sphenoid sinus pneumatization noted was of mixed type.

Diagnostic Nasal Endoscopy was done to confirm the diagnosis and to check for any anatomical abnormality in the nasal cavity and in the sphenoid recess area before proceeding for further management.

According to the recent EPOS 2020 guidelines, Sinusitis associated with Sphenochoanal Polyp is a variant of Non-type 2 Localised Primary Chonic Rhinosinusitis (CRS). EPOS 2020 guidelines clearly state that the management pathway for a localized Primary CRS is primarily a CT scan (after having taken detailed history and clinical assessment) which, if confirms polyposis then it is followed by Surgery.

The patient was planned for Excision of polyp under General anaesthesia after the necessary consent taking $\&$ routine pre-operative investigations. Two $4 \mathrm{~mm}$ nasal endoscopes were used $\left(0^{\circ}\right.$ and $\left.45^{\circ}\right)$. First with the 0 degree endoscope, Diagnostic Nasal Endoscopy was done (Fig. 2) and all three passes were examined in both nasal cavities. 1st Pass in the right nasal cavity showed the polypoidal mass which didn't bleed on touch and was grossly mobile. 2nd pass of right nasal cavity showed the same. 3rd pass of right nasal cavity was normal. The extra sinusal portion of the mass was debrided to expose spheno-ethmoidal recess and the sphenoid ostium. The stalk of the polyp was noted and Sphenoidotomy was done large enough to see the entire intra-sinusal portion of the polyp which was removed along with the stalk subsequently. The Sphenoid ostium was then examined with a $45^{\circ}$ Endoscope to ensure that the mass was fully debrided. 2nd pass was done on the 


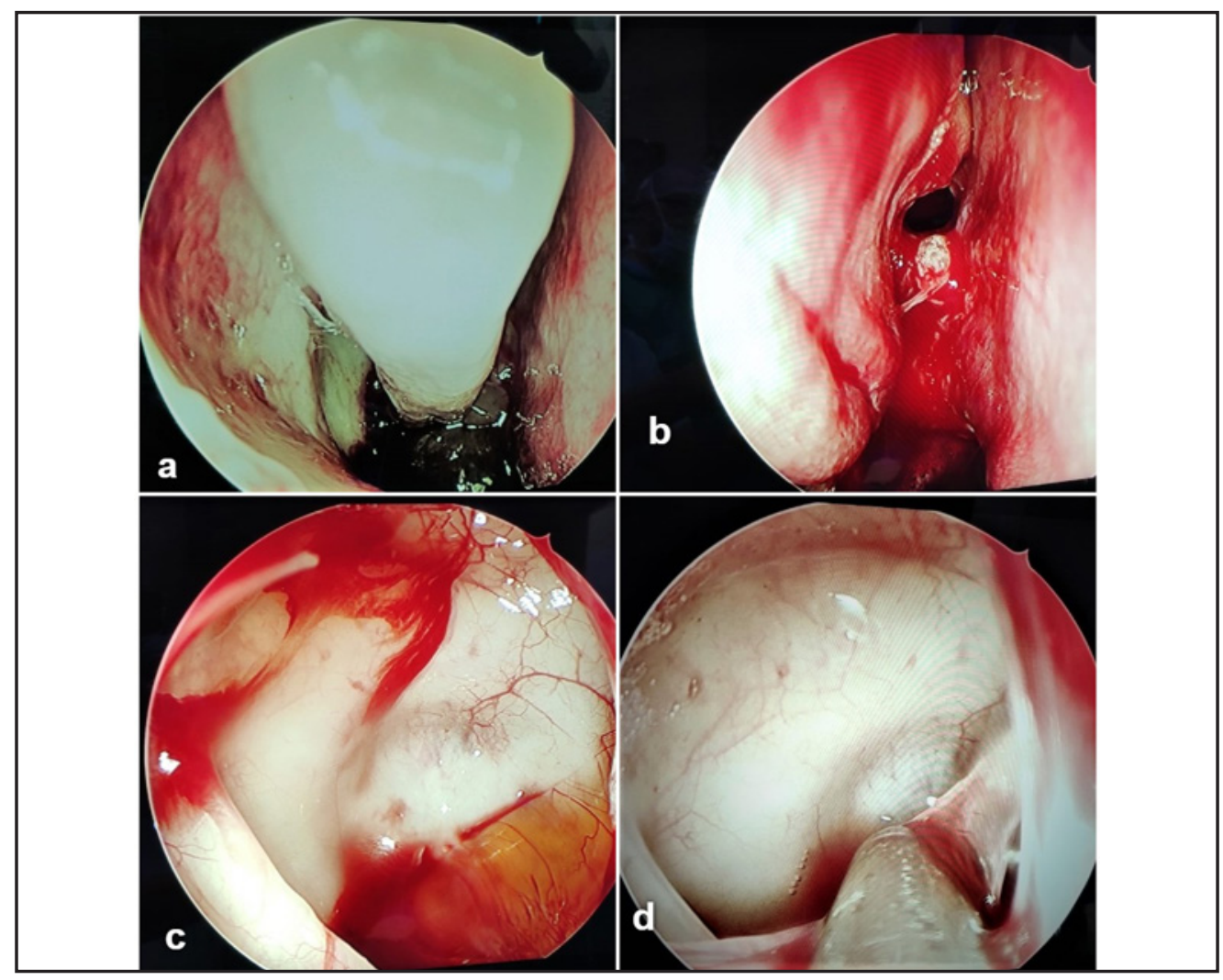

Fig 2. Endoscopic view showing the spheno chonal polyp.

(a) Polyp lying on the right nasal cavity floor. (b) Initial sphenoidotomy( later the opening was made bigger). (c) Intra-sinusal portion of polyp. (d) Sphenoid sinus after excision of polyp.

left side to see any remnant. Haemostasis was achieved and anterior nasal packing was done with merocel. HPE revealed inflammatory polyp with scattered eosinophils. No Malignancy was found.

\section{Discussion}

Sphenochoanal polyp is not very common. On searching the pubmed data base, only 38 articles were noted with isolated sphenochoanal polyp. Spheno-choanal polyp usually occurs in adolescents and young adults. ${ }^{2}$ It is associated with Chronic Rhinosinusitis (CRS) and according to the recent EPOS 2020 guidelines, Primary Chronic Rhinosinusitis has been divided into Localised and Diffuse based on anatomical location. Localised Primary CRS is further divided into Type 2 and Non-type 2 based on endotype dominance. Sinusitis associated with Sphenochoanal Polyp is a variant of Non-type 2 Localised Primary CRS. They are mostly solitary and no gender predilection has been found yet. Etiology of Sphenochoanal polyp is not well known however, according to a study done by Berg et $a 1,{ }^{3}$ they develop from intra mural cyst precursors present in the oedematous mucosa. Allergy too plays minimal role in development of chonal polyps. ${ }^{3}$ Symptoms may vary from patient to patient but common symptoms are nasal obstruction, headache, rhinorrhea, facial pain. ${ }^{3}$

EPOS 2020 guidelines state that the management pathway for a localized Primary CRS is taking the detailed history with performing relevant clinical examination and Diagnostic Nasal Endoscopy followed by a CT scan. CT scan plays a huge role in making further management decisions. If CT scan corroborates with the symptoms of CRS then Surgery is the option to go (Acc 
Table I: Differential Diagnosis of a Sphenochoanal polyp ${ }^{7,8}$

\begin{tabular}{|l|l|}
\hline 1 & Antro-choanal polyp \\
\hline 2 & Adenoid Hypertrophy \\
\hline 3 & Inverted Papilloma \\
\hline 4 & Nasopharyngeal Carcinoma \\
\hline 5 & Thornwaldt's cyst \\
\hline 6 & Lymphoma \\
\hline 7 & Glioma \\
\hline 8 & Hypophyseal tumour \\
\hline 9 & Angiofibroma \\
\hline 10 & Meningoencephalocoele. \\
\hline
\end{tabular}

to EPOS 2020). CT scan not only helps in diagnosis but also helps in planning of surgery. As mentioned above already, CT scan and Diagnostic Nasal Endoscopy together will give a detailed anatomical and pathological status of the patient. The hyper pneumatization of Sphenoid sinus not only allows extended approaches of transsphenoidal surgeries but also increases the likelihood of collision between operative instruments and chances of iatrogenic injuries. ${ }^{1}$ The posterior extension of aeration into the clivus, dorsum sella, and anterior extension into planum sphenoidale may result in an inadvertent damage to the bony walls of the sphenoid sinus, resulting in cerebrospinal fluid (CSF) leaks. ${ }^{4}$ The bony dehiscence and protrusion of the adjacent neurovascular structures into the SS increase in proportion to the pneumatization. ${ }^{5,6}$ The lesser wing extension leads to the protrusion and thinning of bony wall of the optic nerve and internal carotid artery, thereby increasing susceptibility to injury during endoscopic surgeries. ${ }^{6}$ Differential diagnosis is given in Table I.

Surgical excision of the polyp is the preferred treatment and the author insists on certain points which are to be kept in mind while performing surgery. The first being that, if the mass is not found to be what a polyp usually looks like, a biopsy should be considered as safe option prior to excision. Secondly, during excision, proper care has to be taken when the polyp is removed from within the sphenoid sinus so as to avoid injury to the optic nerve and interal carotid artery. And finally, an adequately sized sphenoidotomy to avoid recurrence since it has been seen, that if the polyp is not completely removed, recurrence may occur. ${ }^{9}$

\section{References}

1. Hiremath SB, Gautam AA, Sheeja K, Benjamin G. Assessment of variations in sphenoid sinus pneumatization in Indian population: A multidetector computed tomography study. Indian J Radiol Imaging. 2018;28:273-9

2. Al-Qudah MA. Sphenochoanal polyp: current diagnosis and management. Ear Nose Throat J. 2010; 89: 311-3

3. Berg $\mathrm{O}$, Carenfelt $\mathrm{C}$, Silfverswärd $\mathrm{C}$, Sobin A. Origin of the choanal polyp. Arch Otolaryngol Head Neck Surg. 1988 Nov;114(11):1270-1. doi: 10.1001/ archotol.1988.01860230064025

4. Hamid O, El Fiky L, Hassan O, Kotb A, El Fiky S. Anatomic variations of the sphenoid sinus and their impact on trans sphenoid pituitary surgery. Skull Base 2008; 18:9-15

5. Cho JH, Kim JK, Lee JG, Yoon JH. Sphenoid sinus pneumatization and its relation to bulging of surrounding neurovascular structures. Ann Otol Rhinol Laryngol. 2010;119: 646-50

6. Güldner C, Pistorius SM, Diogo I, Bien S, SesterhennA, WernerJA. Analysis of pneumatization and neurovascular structures of the sphenoid sinus using cone beam tomography (CBT) Acta Radiol. 2012; 53:214-9

7. Soh KBK, Tan KK. Sphenochoanal polyps in Singapore: diagnosis and current management. Singap Med J. 2000; 41:184-7

8. Tosun F, Yetiser S, Akcam T, Ozkaptan Y. Sphenochoanal polyp: endoscopic surgery. Int J Ped Otorhinolaryngol. 2001; 58: $87-90$

9. Bist SS, Kumar R, Varshney S, Bisht M. Isolated sphenochoanal polyp: A rare clinical entity. Indian J Otolaryngol Head Neck Surg. 2007; 59: 56-7. 